Received: 04.06 .2021

Revised: 04.08.2021

Accepted: 27.08 .2021

DOI: $10.17804 / 2410-9908.2021 .4 .050-061$

\title{
INFORMATION AND ANALYTICAL TOOLS FOR ASSESSING THE STRUCTURAL SURVIVABILITY AND SAFETY OF TECHNOLOGICAL EQUIPMENT
}

\author{
E. M. Reizmunt ${ }^{\text {a) }}$, S. V. Doronin ${ }^{\text {b) }}$, and E. V. Moskvichev ${ }^{\text {c) }}$ \\ Federal Research Center for Information and Computational Technologies, \\ 53 Mira Ave., Krasnoyarsk, 660049, Russian Federation \\ a) (iD https://orcid.org/0000-0003-1631-893X ReizmuntEM@ict.nsc.ru; \\ b) (ID https://orcid.org/0000-0002-5256-3871 $\otimes$ sdoronin@ict.nsc.ru; \\ c) iD https://orcid.org/0000-0002-2367-2649 jugr@ict.nsc.ru \\ *Corresponding author. E-mail: ReizmuntEM@ict.nsc.ru \\ Address for correspondence: PO box 25515, Krasnoyarsk, 660000, Russian Federation \\ Tel.: +7 (391) 22772 96; fax: +7 (391) 2124288
}

The tasks of information and computational support for the analysis of the survivability and safety of power shells of technical objects are formulated, aimed at studying their properties taking into account damages and abnormal effects. To solve them, algorithms and computational tools have been developed, aimed at analyzing damage and assessing the risk of re-occurrence of accidents at technical objects, modeling the structural and mechanical heterogeneity of welded joints and calculating their crack resistance, parametric modeling of welded joints with defects, analyzing the sensitivity of structures to defects, damages, and off-design effects, and evaluating their survivability and safety. Information support has been developed, which contains a systematic list of factors reducing survivability and safety, the results of experimental studies of the crack resistance of welded joints and the survivability of shells with a developing macrocrack, as well as the accumulated results of solving model and applied problems on analyzing the properties of damaged shell structures, as reference data.

Keywords: shell structures, survivability, safety, information and computational support.

\section{References}

1. Makhutov N.A., Dukhanina L.N., Svetik F.F., Gadenin M.M., Bobrov Yu.V., Barykin A.N., Zakablutskaya Ye.A., Yudina O.N. Science and technology in the analysis of new challenges and threats in the substantiation of integrated security. In: Bezopasnost Rossii. Pravovye, sotsialnoekonomicheskie i nauchno-tekhnicheskie aspekty. Informatsionnyi byulleten [Safety of Russia: Multivolumed Series]. Moscow, MGOF «Znaniye» Publ., 2021, 152 p. (In Russian).

2. $\quad$ Barishpolets V.A., Bekker A.D., Bobrov Yu.V., Vlasov Yu.V., Gadenin M.M., Korchak V.Yu., Krivopuskov V.V., Litvinov V.B., Makhutov N.A., Reznikov D.O., Yudin A.Yu. Bezopasnost Rossii: Tematicheskiy blok Natsionalnaya bezopasnost [Safety of Russia: Thematic Topic "National Safety"]. Moscow, MGOF «Znanie» Publ., 2021, 432 p. ISBN 978-5-87633-195-3. (In Russian).

3. Makhutov N.A., Cherepanov A.P., Lisanov M.V. Tasks of the development and implementation of enterprise industrial safety management system when operating technical devices. $\mathrm{Be}$ zopasnost truda $v$ promyshlennosti, 2021, No. 2, pp. 15-19. DOI 10.24000/0409-2961-2021-2-1519. (In Russian).

4. Mahutov N.A., Reznikov D.O. Multilevel Survivability Assessment of Complex Technical Systems with Account for Large-Scale and Structural Hierarchy of Damages and Destruction Accumulation Processes. Safety in Technosphere, 2016, vol. 5, iss. 4, no. 4, pp. 3-17. (In Russian). 
5. Makhutov N.A., Reznikov D.O., Petrov P.V. Assessment of survivability of complex technical systems. Problemy Bezopasnosti i Chrezvychaynykh Situatsiy, 2009, no. 3. pp. 47-66. (In Russian).

6. Lepikhin A.M., Makhutov N.A., Shokin Yu.I. Probabilistic multiscale modeling of fracture in heterogeneous materials and structures. Industrial laboratory. Diagnostics of materials, 2020, 86 (7), pp. 45-54. DOI: 10.26896/1028-6861-2020-86-7-45-54. (In Russian).

7. Lepikhin A.M., Makhutov N.A., Shokin Yu.I., Yurchenko A.V. Analysis of risk concept for technical systems using digital twins. Vychislitelnye Tekhnologii, 2020, no. 4, pp. 99-113. DOI: 10.25743/ICT.2020.25.4.009. (In Russian).

8. Makhutov N.A., Gadenin M.M., Maslov S.V., Reznikov D.O., Pichkov S.N., Panov V.A. Investigation of the Influence of Operational Loading Regimes on the Service Life of Nuclear Power Plants. In: H. Altenbach, V.A. Eremeyev, L.A. Igumnov, eds. In: Multiscale Solid Mechanics, Advanced Structured Materials series, Springer, 2021, vol 141, pp. 319-330. DOI: 10.1007/978-3-030-54928-2_24.

9. Makhutov N.A., Gadenin M.M., Reznikov D.O. Assessment of extreme thermo-mechanical states of engineering systems under operating loading conditions. Acta Mechanica. 2021. vol. 232, No. 5, pp. 1829-1839. DOI 10.1007/s00707-020-02920-3.

10. Makhutov N.A., Lepikhin A.M., Leshchenko V.V. Scientific and methodological provision of safety of subsea pipelines with defects using risk criteria. Zavodskaya laboratoriya. Diagnostika materialov, 2021, vol. 87. no. 6, pp. 45-53. DOI 10.26896/1028-6861-2021-87-6-45-53. (In Russian).

11. Makhutov N.A., Panov A.N. Improving the scientific base and regulatory justification of the safety of wheeled mobile vehicles. Problemy Bezopasnosti i Chrezvychaynykh Situatsiy, 2021, no. 1, pp. 34-43. DOI 10.36535/0869-4176-2021-01-5. (In Russian).

12. Makhutov N.A., Makarenko I.V., Makarenko L.V. Features of an estimation low temperatures of crack resistance for cryogenic techniques. In: Zhivuchest $i$ konstruktsionnoye materialovedeniye (ZhiVKoM-2020) [The $5^{\text {th }}$ International Scientific and Technical on line Conference "Survivability and Structural Science of Materials": collection of papers]. Moscow, October 27-29 2020, Moscow, IMASH im. A.A. Blagonravova RAN Publ., 2020, pp. 154-157. (In Russian).

13. Zhuravkov M.A., Bogdanovich A.V., Shemet L.A., Nikolaychik M.A., Dembovsky I.N., Makhutov N.A., Gadenin M.M., Reznikov D.O., Yudina O.N. Modelling of contact interaction between parts of the lifting mine complex at safety estimations. Problemy bezopasnosti i chrezvychaynykh situatsiy, 2021, No. 4, pp. 9-25. DOI: 10.36535/0869-4179-2021-04-1. (In Russian).

14. Makhutov N.A., Nadein V.A., Blednova Zh.M., Neganov D.A., Shauro A.N. Problems of substantiating the strength and safety of main pipelines, taking into account the stages of the life cycle, hazardous technogenic and natural factors. Problemy bezopasnosti i chrezvychaynykh situatsiy, 2021, no. 1, pp. 5-17. DOI: 10.36535/0869-4176-2021-01-1. (In Russian).

15. Makhutov N.A., Kossov V.S., Oganyan E.S., Volokhov G.M., Ovechnikov M.N., Protopopov A.L. Prediction of contact-fatigue damage to rails using computational-experimental methods. Zavodskaya laboratoriya. Diagnostika materialov, 2020, vol. 86, No. 4, pp. 46-55. DOI: 10.26896/1028-6861-2020-86-4-46-55. (In Russian).

16. Makhutov N.A. Management of safety of critical and strategically important marine mobile facilities according to risk creation. In: Sb. tr. konf. "Upravlenie $v$ morskikh sistemakh" (UMS -2020): 13-ya Mul'tikonferentsiya po problemam upravleniya [Proceedings of the "Management of Maritime systems" (UMS-2020)], St. Petersburg, October 07-08 2020, St. Petersburg, Kontsern "CNII “Elektropribor” Publ., 2020, pp. 21-22. (In Russian). 
17. Moskvichev E.V., Lepihin A.M. Structural and mechanical heterogeneity and fracture toughness of welded joints of steels 09Mn2Si and 12Cr18Ni10Ti. Zavodskaya laboratoriya. Diagnostika materialov, 2013, no 6, pp. 50-54. (In Russian).

18. Rogalev A.N., Doronin S.V., Reizmunt E.M. Experience in solving and formulating inverse problems of structural strength and survivability. Trudy mezhdunarodnoy konferentsii "Aktualnyye problemy vychislitelnoy i prikladnoy matematiki-2015", posvyashchennoy 90-letiyu so dnya rozhdeniya akademika Guriya Ivanovicha Marchuka [Proceedings of International Conference dedicated to $90^{\text {th }}$ anniversary of G.I. Marchuk's birth “Actual problems of computational and applied mathematics-2015"], Novosibirsk, Abvey, 19-23 October 2015, 916 p. (In Russian).

19. Moskvichev E.V. An evaluation of the fracture toughness of a thin-walled pressure vessel with account for the structural and mechanical inhomogeneity of the welded joint. J. Mach. Manuf. Reliab., 2015, vol. 44, pp. 32-37. DOI: 10.3103/S1052618815020119.

20. Moskvichev E. Fracture assessment of cracked welded structures considering the heterogeneity of welded joints. Procedia Materials Science, 2014, vol. 3, pp. 556-561. DOI: 10.1016/j.mspro.2014.06.092. 21. Larionov V.P., Moskvichev V.V., Doronin S.V. Fracture toughness analysis of typical welded joints of excavator metal structures. Sibirskiy fiziko-tekhnicheskiy zhurnal, 1993, vol. 2, pp. 104-113 (In Russian).

22. Shokin Yu.I., Moskvichev V.V., Lepihin A.M. Veroyatnostnyye modeli tekhnologicheskoy defektnosti svarnykh soyedineniy [Probabilistic models of technological defects in welded joints]. Krasnoyarsk, VTs SO AN SSSR Publ., 1988. (In Russian).

23. Moskvichev V.V., Doronin S.V. Assessment and optimization of durability and reliability in the life design of welded structures. Zavodskaya laboratoriya. Diagnostika materialov, 1996, no 3, pp. 38-42. (In Russian).

24. Stoer M., Wagner F. A simple min-cut algorithm. Journal of the ACM, 1997, vol. 44, no. 4, pp. 585-591. DOI: 10.1145/263867.263872. 
Подана в журнал: 04.06.2021

УДК 62-19:621.45.017

DOI: $10.17804 / 2410-9908.2021 .4 .050-061$

\title{
ИНФОРМАЦИОННО-АНАЛИТИЧЕСКИЕ СРЕДСТВА ОЦЕНКИ КОНСТРУКЦИОННОЙ ЖИВУЧЕСТИ И БЕЗОПАСНОСТИ ТЕХНОЛОГИЧЕСКОГО ОБОРУДОВАНИЯ
}

\author{
Е. М. Рейзмунт ${ }^{\text {a)* }}$, С. В. Доронин ${ }^{\text {()) }}$ Е. В. Москвичев ${ }^{\text {в) }}$ \\ Федеральный исследовательский иентр информационных и вычислительных технологий, \\ 2. Красноярск, Российская Федераџия \\ а) iD https://orcid.org/0000-0003-1631-893X ReizmuntEM@ict.nsc.ru;
б) (iD https://orcid.org/0000-0002-5256-3871 sdoronin@ict.nsc.ru;
в) iD https://orcid.org/0000-0002-2367-2649 jugr@ict.nsc.ru \\ *Ответственный автор. Электронная почта: ReizmuntEM@ict.nsc.ru \\ Адрес для переписки: a/я 25515, г. Красноярск, 660000, Российская Федерация, \\ Тел.: +7 (391) 227-72-96; факс: +7 (391) 212-42-88
}

Сформулированы задачи информационно-вычислительного обеспечения анализа живучести и безопасности силовых оболочек технических объектов, направленные на изучение их свойств с учетом повреждений и нештатных воздействий. Для их решения разработаны алгоритмы и вычислительные средства, направленные на анализ аварий и оценку риска их повторного возникновения, моделирование структурно-механической неоднородности и расчет трещиностойкости сварных соединений, параметрическое моделирование сварных соединений с дефектами, анализ чувствительности конструкций к дефектам, повреждениям и запроектным воздействиям, оценку живучести и безопасности технологического оборудования (ТО). Разработано информационное обеспечение, содержащее в качестве справочных данных систематизированный перечень факторов снижения живучести и безопасности ТО, результаты экспериментальных исследований трещиностойкости сварных соединений и живучести оболочек с развивающейся макротрещиной, накопленные результаты решения модельных и прикладных задач анализа свойств повреждаемых оболочечных конструкций.

Ключевые слова: оболочечные конструкции, живучесть, безопасность, информационновычислительное обеспечение.

\section{1. Введение}

Исследования общих вопросов анализа и обеспечения техногенной безопасности [1-3] на всех иерархических уровнях элементов инфраструктуры формируют цели и задачи в области конструкционной прочности и живучести отдельных типов и классов технических систем, комплексов объектов, инженерных сооружений, машин и оборудования. Они касаются как общих методических вопросов анализа конструкционной прочности и живучести [4-7], так и технических объектов с учетом их отраслевой специфики [8-16]. В статье рассмотрены возможности практического получения количественных оценок живучести и безопасности технологического оборудования, преимущественно состоящего из сварных оболочечных конструкций.

Анализ конструкционной живучести и безопасности технологического оборудования предполагает получение количественных оценок характеристик, описывающих поведение конструкций в поврежденном состоянии и в условиях запроектного нагружения. В силу этого актуально развитие как информационного обеспечения, так и инструментальных средств оценивания и анализа живучести и безопасности. 
Информационное обеспечение анализа живучести и безопасности технологического оборудования дает возможность использовать следующие справочные данные:

- систематизированный перечень факторов снижения живучести и безопасности ТО;

- результаты экспериментальных исследований трещиностойкости сварных соединений и живучести элементов конструкций с развивающейся макротрещиной;

- накопленные результаты решения модельных и прикладных задач анализа свойств повреждаемых конструкций ТО.

\section{2. Описание информационно-аналитической системы}

Систематизация факторов снижения живучести и безопасности направлена на информационное обеспечение анализа уязвимостей, формирующих предпосылки к повреждению и разрушению силовых конструкций. Рассмотрены конструктивные (особенности, порождающие сингулярность численных решений; фактически неудачные технические решения, выявленные при эксплуатации конструкций) и технологические (нарушения технологических процессов сварки и технологии монтажа) факторы.

Описание конструктивных факторов включает эскизы, чертежи, схемы, фотографии, иллюстрирующие неудачные конструктивные решения, последствия в виде зон трещинообразования и разрушения.

Описание технологических факторов представлено в табличной форме, и в виде правил логического вывода вида ЕСЛИ <Предпосылка $>$ ТО $<$ Заключение >, в которых предпосылками являются события, процессы, явления, приводящие к нарушению технологических правил сварки, а заключениями - негативные последствия, приводящие к снижению живучести и безопасности технических объектов.

Результаты экспериментальных исследований механических свойств и трещиностойкости сварных соединений представлены средними значениями и коэффициентами вариации предела текучести, временного сопротивления и $J$-интеграла сталей 09Г2С и $12 \mathrm{X} 18 \mathrm{H} 10 \mathrm{~T}$, широко используемых в современном машиностроении для изготовления сосудов, аппаратов, емкостей, трубопроводов и других объектов, работающих под давлением (таблица).

Средние значения $\bar{x}$ и коэффициенты вариации н механических свойств и $J$-интеграла в зонах сварного соединения [17]

\begin{tabular}{|c|c|c|c|c|c|c|}
\hline \multirow{2}{*}{ Зона сварного шва } & \multicolumn{2}{|c|}{ Предел текучести } & \multicolumn{2}{|c|}{ Временное сопротивление } & \multicolumn{2}{|c|}{$J$-интеграл } \\
\hline & $\bar{x}, \mathrm{MПа}$ & $\mathrm{H}$ & $\bar{x}, \mathrm{MПа}$ & $\mathrm{H}$ & 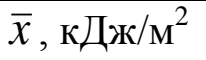 & $\mathrm{H}$ \\
\hline \multicolumn{7}{|c|}{$09 \Gamma 2 \mathrm{C}$} \\
\hline Основной металл & 442 & 0,04 & 593 & 0,02 & 84 & 0,14 \\
\hline Зона термовлияния & 466 & 0,07 & 631 & 0,01 & 87 & 0,26 \\
\hline Металл шва & 414 & 0,06 & 571 & 0,04 & 89 & 0,07 \\
\hline \multicolumn{7}{|c|}{$12 \mathrm{X} 18 \mathrm{H} 10 \mathrm{~T}$} \\
\hline Основной металл & 397 & 0,07 & 686 & 0,004 & 481 & 0,05 \\
\hline Зона термовлияния & 406 & 0,05 & 668 & 0,02 & 545 & 0,19 \\
\hline Металл шва & 420 & 0,16 & 661 & 0,07 & 495 & 0,04 \\
\hline
\end{tabular}

Справочные результаты экспериментальных исследований живучести типовых оболочечных элементов конструкций с макроскопическими трещинами содержат характерные траектории развития усталостных трещин, которые могут быть использованы для прогнозных оценок долговечности оболочечных конструкций. Систематизация результатов решения задач анализа повреждаемых конструкций предполагает структурирование и представление данных в виде следующих информационных единиц:

- описание технического объекта; 
- описание проблемной ситуации (рис. 1);

- исходные данные для постановки исследования;

- используемые характеристики живучести и безопасности, унифицированные постановки задач исследования;

- особенности постановки задач и основные результаты.

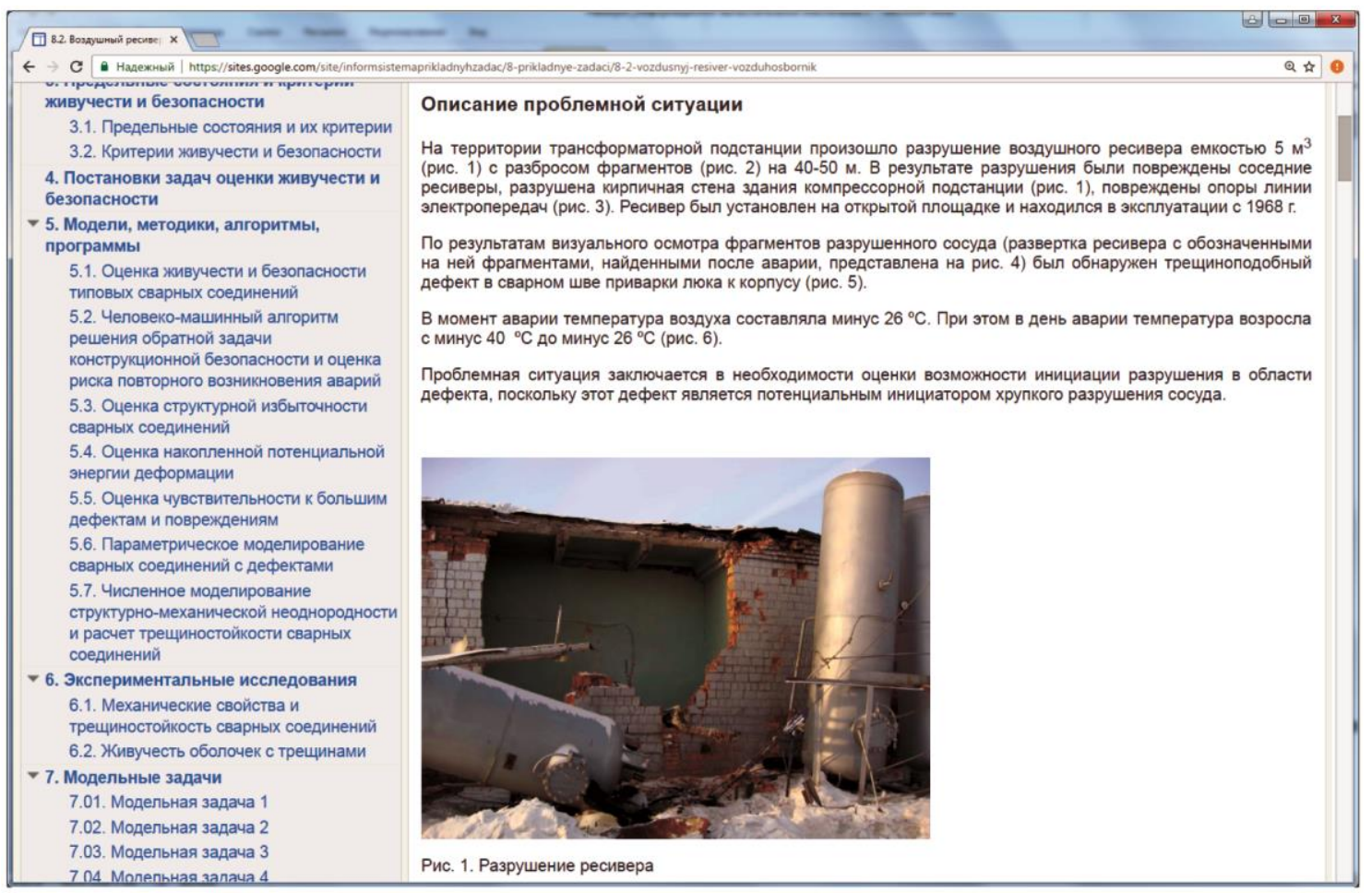

Рис. 1. Описание проблемной ситуации в информационной системе

В развитии инструментальных средств преобладает разработка оригинальных прогностических процедур, вычислительных алгоритмов и программного обеспечения, направленных на реализацию следующих функций:

- анализ аварийных разрушений и оценка риска повторного возникновения аварий технических объектов;

- моделирование структурно-механической неоднородности и расчет трещиностойкости сварных соединений;

- параметрическое моделирование сварных соединений с дефектами;

- анализ чувствительности конструкций к дефектам, повреждениям и запроектным воздействиям;

- оценка живучести и безопасности, структурной избыточности, потенциальной энергии деформации сварных соединений.

Анализ аварийных разрушений осуществляется с использованием человеко-машинного алгоритма решения обратных задач [18], включающего исследование особенностей напряженного состояния конструкций в нештатных ситуациях и при различных вариантах развития аварии; разработку структурных моделей силовых конструкций, позволяющих установить закономерности разрушения силовых конструкций; моделирование причинно-следственных связей, приводящих к последовательным разрушениям. При постановке обратных задач в качестве следствия рассматриваются фактические повреждения и разрушения, а в качестве причин - нерасчетные (завышенные) нагрузки и воздействия произвольной природы, нарушение граничных условий (проектных условий взаимодействия с окружающими элементами 
природной среды и техносферы), наличие дефектов (преимущественно сплошности материала и сборки конструкции).

Моделирование структурно-механической неоднородности и расчет трещиностойкости сварных соединений обеспечиваются разработанным алгоритмом, реализованным в виде макроса в среде конечно-элементного моделирования ANSYS, написанным на языке APDL (ANSYS Parametric Design Language). Согласно алгоритму конечно-элементные модели сварных соединений учитывают стохастический характер распределения механических свойств в различных зонах сварного шва. Материал сварного соединения представляется совокупностью малых структурных элементов со случайными значениями предела текучести и временного сопротивления, изменяющимися в различных зонах сварного шва согласно некоторому закону распределения $[19,20]$. Таким образом, каждый структурный элемент сварного соединения обладает собственной диаграммой деформирования.

При расчете на трещиностойкость рассматриваемой модели сварного соединения в упругопластической постановке используется энергетический критерий, основанный на концепции $J$-интеграла. Это позволяет учитывать нелинейность процессов деформирования, а также распределение механических свойств не только в вершине, но и в окрестности трещины.

Параметрическое моделирование сварных соединений с дефектами позволяет при отсутствии достоверных данных относительно геометрических характеристик и зон локализации дефектов и повреждений получить интервальные оценки напряженнодеформированного состояния элементов конструкций с дефектами и повреждениями, широко используемые при анализе их живучести и безопасности. Неопределенность снижается посредством автоматизации многовариантного численного анализа с варьированием геометрических характеристик поврежденных зон с целью поиска наихудших с позиций живучести и безопасности результатов.

На языке APDL разработан макрос построения и анализа параметрической модели конструкции патрубкового узла цилиндрического сосуда давления с эллиптическими днищами. В качестве обобщенной модели дефекта типа «пора» и «включение» рассматривается несплошность в виде эллипсоида вращения, варьирование соотношения величин осей которого позволяет в широком диапазоне моделировать геометрию дефектов. Геометрия сосуда и патрубкового узла с дефектом сварного шва описывается множеством параметров, из которых одни (внутренний радиус обечайки сосуда; соотношение осей эллиптического днища; толщина стенки сосуда; высота цилиндрической обечайки сосуда; внутренний радиус патрубка; толщина стенки патрубка; давление; катет шва) формируют геометрию и нагружение узла, а другие (большая и малая оси эллиптического дефекта; параметры локализации дефекта в сечении сварного шва) - размер и положение дефекта. В автоматическом режиме по результатам анализа определяется интенсивность распределения напряжений по Мизесу в области локализации дефекта. Доступны для анализа и другие характеристики напряженнодеформированного состояния.

Анализ чувствительности конструкций к дефектам, повреждениям и запроектным воздействиям предполагает отслеживание отклика системы на малое воздействие извне (увеличение нагрузки) либо деградационные изменения внутри конструкции (развитие дефекта или повреждения). Применительно к оценке живучести силовых конструкций с дефектами, повреждениями и в условиях запроектных воздействий в качестве такого малого воздействия предлагается рассматривать единичное приращение размера дефекта $d a$ либо величины нагрузки $d P$, а в качестве реакции - результирующее изменение пиковых значений интенсивности напряжений $d y$. Тогда в качестве оценки чувствительности рассматривается отношение $d \mathrm{y}$ к $d a(d P)$ как функция размера дефекта или повреждения (нагружения) $d \mathrm{y} / d a=f(a)$ $(d y / d P=f(P))$. Такая количественная оценка чувствительности не является критериальной характеристикой, поскольку отсутствует достоверная база для сравнительного анализа. 
Оценки чувствительности, получаемые для различных конструктивных зон одного или нескольких объектов, позволяют судить об уровне живучести с использованием качественных характеристик: «очень высокая», «высокая», «средняя», «низкая», «очень низкая».

Оценка живучести и безопасности типовых сварных соединений основана на алгоритме расчета циклической трещиностойкости сварных соединений с трещиноподобным дефектом [21]. В соответствии со статистическими исследованиями описание дефекта осуществляется в вероятностной постановке в предположении Вейбулловского распределения его размеров [22]. Алгоритм реализует метод статистических испытаний (Монте-Карло) для моделирования кинетики трещины в сварном соединении. Для его практического использования разработано приложение для Windows (рис. 2). На основании прогнозируемых статистических оценок циклической долговечности выполняется построение полных вероятностных диаграмм живучести [23], связывающих характеристики вероятности, долговечности с уровнями нагруженности и поврежденности, характеризуемой размерами и локализацией трещиноподобного дефекта.

Наличие и степень структурной избыточности могут быть оценены с использованием графовых моделей структуры сварной оболочечной конструкции, в которых элементы (детали) представляются вершинами графа, а сварные швы - его ребрами (дугами). Структурная избыточность, наличие нескольких путей передачи силового потока являются факторами, способствующими повышению живучести ТО. При анализе связей элементов в рамках структурной схемы выявляем, приводит ли разрыв связи к потере конструкционной целостности или сохраняются альтернативные пути передачи силового потока. В этом случае оценки живучести носят качественный характер и отвечают на вопрос: обладает ли конструкция свойством живучести (имеются избыточные структурные связи) или нет (избыточных связей нет и разрыв любой из них означает нарушение конструкционной целостности).

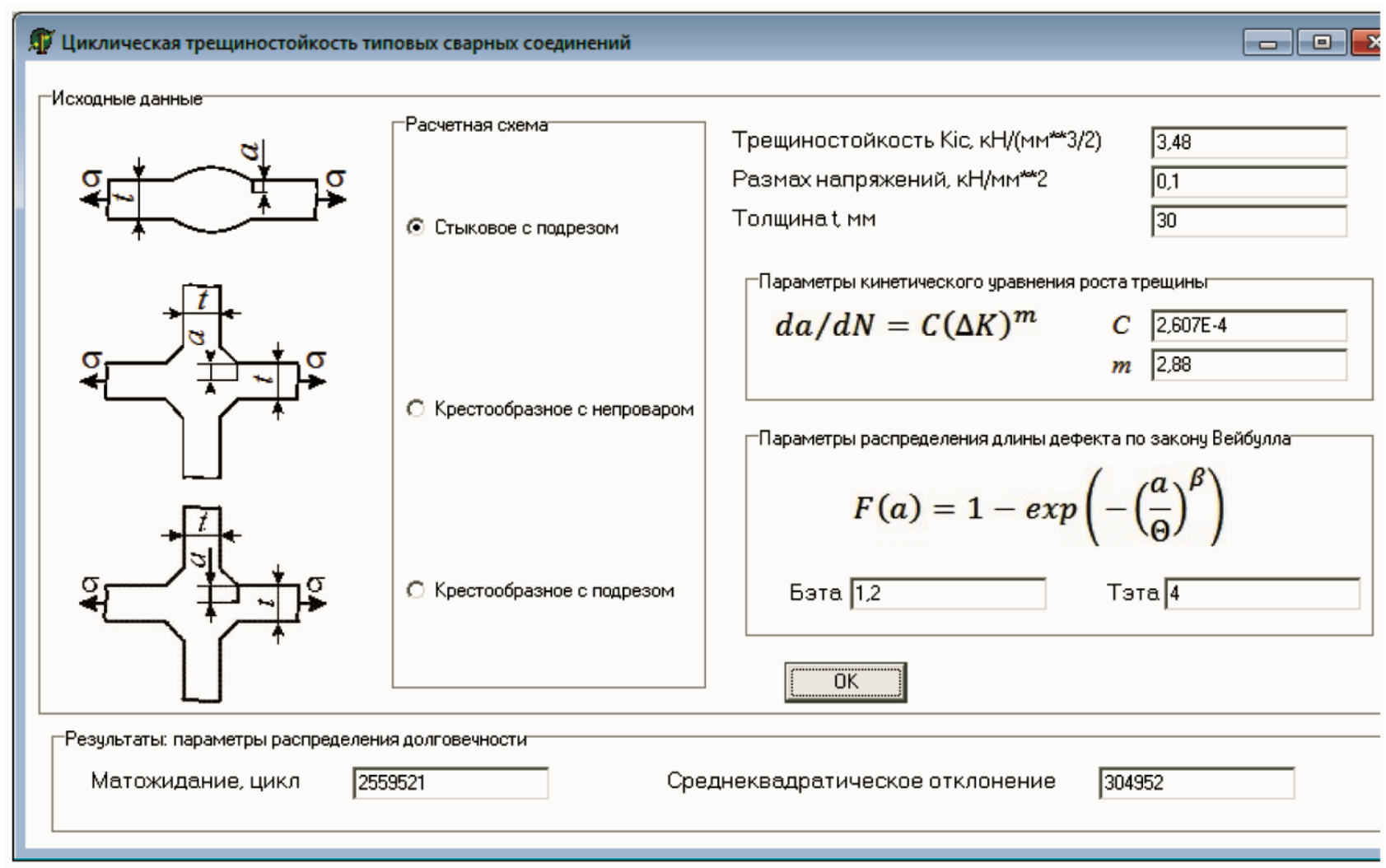

Рис. 2. Интерфейс программы расчета типовых сварных соединений с дефектами 
Оценка структурной избыточности сварных соединений реализуется с помощью следующего алгоритма:

1) анализ сварной оболочечной конструкции и построение ее структурной модели;

2) визуализация графа и построение его матрицы смежности;

3) вычисление минимального разреза графа $\mathrm{n}_{\min } \mathrm{c}$ помощью разработанного приложения, реализующего алгоритм Штор-Вагнера [24];

4) оценка структурной избыточности сварных соединений $\mathrm{n}_{\min }-1$.

Уровень накопленной в конструкции потенциальной энергии деформации является одним из факторов, формирующих ее техногенную опасность. Это вытекает из превращения энергии деформации как в энергию, затрачиваемую на разрушение, так и в кинетическую энергию элементов конструкции при нарушении ее целостности. Очевидно, увеличение уровня накопленной потенциальной энергии деформации сопровождается снижением безопасности эксплуатации технического объекта.

Несмотря на то, что количественная оценка накопленной энергии деформации не вызывает затруднений, существует неопределенность относительно критических (соответствующих опасному состоянию объекта) значений потенциальной энергии деформации. Таким образом, можно говорить о применении оценок потенциальной энергии деформации в качестве нечеткой характеристики безопасности.

В общем случае для оценки накопленной в объеме материала потенциальной энергии деформации используется выражение

$$
u=\frac{1}{2} \int_{V}\left(\sigma_{1} \varepsilon_{1}+\sigma_{2} \varepsilon_{2}+\sigma_{3} \varepsilon_{3}\right) d V,
$$

для дискретной (конечно-элементной) модели размерности $N$, принимающее вид

$$
u=\frac{1}{2} \sum_{1}^{N}\left(\sigma_{1} \varepsilon_{1}+\sigma_{2} \varepsilon_{2}+\sigma_{3} \varepsilon_{3}\right)
$$

Вычислительная технология оценки накопленной в объеме материала сварного шва потенциальной энергии деформации, отнесенной к единице его длины, реализована в среде конечно-элементного анализа с применением макроса на языке APDL и включает следующие основные этапы: ной модели;

1) описание сварных швов самостоятельными объемами при разработке твердотель-

2) выделение на стадии расчета НДС конечных элементов, ассоциированных с объемами сварных швов;

3) вычисление величины потенциальной энергии деформации, накопленной в каждом $i$-м $(i=1, N)$ конечном элементе;

4) организацию цикла суммирования по объему каждого сварного шва величин потенциальной энергии деформации, накопленной в каждом конечном элементе;

5) отнесение накопленной в объеме сварного шва потенциальной энергии деформации к единице его длины.

\section{3. Результаты и обсуждение}

Разработанные информационно-аналитические средства реализуют системный подход к постановке и решению задач анализа живучести и безопасности технических объектов, содержащих силовые оболочечные конструкции. 
Наибольшая эффективность информационно-вычислительного ресурса достигается при следующей технологии последовательного использования его составных частей:

- качественный анализ наличия конструктивных и технологических факторов снижения живучести и безопасности с целью идентификации уязвимостей и обоснования рекомендаций по их устранению;

- выбор одного или нескольких предельных состояний, возможных для рассматриваемого объекта;

- выбор предлагаемых критериев живучести и безопасности, соответствующих принятым предельным состояниям, структуре и свойствам технического объекта;

- анализ систематизированных возможных постановок задач живучести и безопасности рассматриваемого технического объекта с целью свести его к одной или нескольким унифицированным постановкам модельных и прикладных задач. При этом целесообразен анализ накопленного практического опыта решения этих задач;

- использование для оценки выполнения критериев живучести и безопасности как общетехнических методов, так и разработанных в рамках проекта специализированных методик, алгоритмов, программ;

- использование при получении оценок живучести и безопасности экспериментальных данных по механическим свойствам сталей, условиям инициации и распространения трещин в элементах оболочечных конструкций.

\section{4. Заключение}

Разработанные информационное обеспечение и инструментальные средства интегрированы в информационную систему «Живучесть и безопасность оболочечных конструкций технических объектов», реализованную в виде Web-приложения: https://sites.google.com/site/ /informsistemaprikladnyhzadac/

Накопление результатов решения задач анализа повреждаемых конструкций обеспечивает научно-методическое обеспечение научно-технической экспертизы аварийных разрушений и техногенных катастроф.

\section{Литература}

1. Наука и технологии в проблемах анализа новых вызовов и угроз при обосновании комплексной безопасности : итоговые материалы по I-II этапам и перспективам реализации многотомного издания «Безопасность России. Правовые, социально-экономические и научно-технические аспекты : информационный бюллетень» / Н. А. Махутов, Л. Н. Духанина, Ф. Ф. Светик, М. М. Гаденин, Ю. В. Бобров, А. Н. Барыкин, Е. А. Закаблуцкая, О. Н. Юдина. Москва : МГОФ «Знание», 2021. - 152 с. - ISBN 978-5-87633-196-0.

2. Безопасность России. Правовые, социально-экономические и научно-технические аспекты. Безопасность сложных человеко-машинных систем : тематический блок «Национальная безопасность» / В. А. Баришполец, А. Д. Беккер, Ю. В. Бобров, Ю. В. Власов, М. М. Гаденин, В. Ю. Корчак, В. В. Кривопусков, В. Б. Литвинов, Н. А. Махутов, Д. О. Резников, А. Ю. Юдин. - Москва : МГОФ «Знание», 2021. - 432 с. - ISBN 978-5-87633-195-3. 3. Махутов Н. А., Черепанов А. П., Лисанов М. В. Задачи разработки и реализации системы управления промышленной безопасностью предприятия при эксплуатации технических устройств // Безопасность труда в промышленности. - 2021. - № 2. - С. 15-19. DOI: 10.24000/0409-2961-2021-2-15-19.

4. Махутов Н. А., Резников Д. О. Многоуровневая оценка живучести сложных технических систем с учетом масштабно-структурной иерархии процессов накопления повреждений и разрушения // Безопасность в техносфере. - 2016. - № 4. - С. 3-17.

5. Махутов Н. А., Резников Д. О. Оценка прочностной живучести технических систем // Проблемы безопасности и чрезвычайных ситуаций. - 2019. - № 3. - С. 47-57. 
6. Лепихин А. М., Махутов Н. А., Шокин Ю. И. Вероятностное многомасштабное моделирование разрушений структурно-неоднородных материалов и конструкций // Заводская лаборатория. Диагностика материалов. - 2020. - № 67. - С. 45-54.

7. Концепция риск-анализа технических систем с использованием цифровых двойников / А. М. Лепихин, Н. А. Махутов, Ю. И. Шокин, А. В. Юрченко // Вычислительные технологии. - 2020. - № 4. - C. 99-113.

8. Investigation of the influence of operational loading regimes on the service life of nuclear power plants / N. A. Makhutov, M. M. Gadenin, S. V. Maslov, Dmitry O. Reznikov, Sergey N. Pichkov, Vladimir A. Panov // Multiscale solid mechanics : strength, durability, and dynamics. Switzerland : Springer Nature, 2021. - P. 319-330. - DOI: 10.1007/978-3-030-54928-2_24.

9. Makhutov N. A., Gadenin M. M., Reznikov D. O. Assessment of extreme thermomechanical states of engineering systems under operating loading conditions // Acta Mechanica. 2021. - Vol. 232, No 5. - P. 1829-1839. - DOI 10.1007/s00707-020-02920-3.

10. Махутов Н. А., Лепихин А. М., Лещенко В. В. Научно-методическое обеспечение безопасности морских подводных трубопроводов с дефектами по критериям риска // Заводская лаборатория. Диагностика материалов. - 2021. - Т. 87, № 6. - С. 45-53. DOI 10.26896/1028-6861-2021-87-6-45-53.

11. Махутов Н. А., Панов А. Н. Совершенствование научной базы и нормативного обоснования безопасности колесных мобильных машин // Проблемы безопасности и чрезвычайных ситуаций. - 2021. - № 1. - С. 34-43. - DOI 10.36535/0869-4176-2021-01-5.

12. Махутов Н. А., Макаренко И. В., Макаренко Л. В. Особенности оценки низкотемпературной трещиностойкости для криогенной техники // Живучесть и конструкционное материаловедение (ЖивКоМ-2020) : сборник трудов V Международной научно-технической конференции в дистанционном формате, Москва, 27-29 октября 2020 года. - Москва : Федеральное государственное бюджетное учреждение науки Институт машиноведения им. А. А. Благонравова Российской академии наук, 2020. - С. 154-157.

13. Моделирование контактного взаимодействия между элементами подъемного шахтного комплекса при оценках безопасности / М. А. Журавков, А. В. Богданович, Л. А. Шемет, М. А. Николайчик, И. Н. Дембовский, Н. А. Махутов, М. М. Гаденин, Д. О. Резников, О. Н. Юдина // Проблемы безопасности и чрезвычайных ситуаций. - 2021. - № 4. - С. 9-25.

14. Проблемы обоснования прочности и безопасности магистральных трубопроводов с учетом стадий жизненного цикла, опасных техногенных и природных факторов / Н. А. Махутов, В. А. Надеин, Ж. М. Бледнова, Д. А. Неганов, А. Н. Шауро // Проблемы безопасности и чрезвычайных ситуаций. - 2021. - № 1. - С. 5-17.

15. Прогнозирование контактно-усталостных повреждений рельсов расчетноэкспериментальными методами / Н. А. Махутов, В. С. Коссов, Э. С. Оганьян, Г. М. Волохов, М. Н. Овечников, А. Л. Протопопов // Заводская лаборатория. Диагностика материалов. 2020. - Т. 86, № 4. - С. 46-55. - DOI 10.26896/1028-6861-2020-86-4-46-55.

16. Махутов Н. А. Управление безопасностью по критериям рисков критически и стратегически важных морских подвижных объектов // Материалы конференции «Управление в морских системах» (УМС-2020) : 13-я МУЛЬТИКОНФЕРЕНЦИЯ ПО ПРОБЛЕМАМ УПРАВЛЕНИЯ, Санкт-Петербург, 07-08 октября 2020 года. - Санкт-Петербург, Концерн Центральный научно-исследовательский институт «Электроприбор», 2020. - С. 21-22.

17. Москвичев Е. В., Лепихин А. М. Структурно-механическая неоднородность и трещиностойкость сварных соединений сталей 09Г2С и 12Х18Н10Т // Заводская лаборатория. Диагностика материалов. - 2013. - № 6. - С. 50-54.

18. Рогалев А. Н., Доронин С. В., Рейзмунт Е. М. Опыт решения и постановки обратных задач конструкционной прочности и живучести [Электронный ресурс] // Труды международной конференции «Актуальные проблемы вычислительной и прикладной математики-2015», посвященной 90-летию со дня рождения академика Гурия Ивановича Марчука, Абвей, 19-23 
октября 2015 г. - Институт вычислительной математики и математической геофизики Сибирского отделения Российской академии наук, Новосибирск, 2015. - 1 электрон. опт. диск. -916 с.

19. Moskvichev E. V. An evaluation of the fracture toughness of a thin-walled pressure vessel with account for the structural and mechanical inhomogeneity of the welded joint // J. Mach. Manuf. Reliab. - 2015. - Vol. 44. - P. 32-37 - DOI 10.3103/S1052618815020119.

20. Moskvichev E. Fracture assessment of cracked welded structures considering the heterogeneity of welded joints // Procedia Materials Science. - 2014. - Vol. 3. - P. 556-561 DOI 10.1016/j.mspro.2014.06.092.

21. Ларионов В. П., Москвичев В. В., Доронин С. В. Расчет на трещиностойкость типовых сварных соединений металлоконструкций экскаваторов // Сибирский физико-технический журнал. - 1993. - Вып. 2. - С. 104-113.

22. Шокин Ю. И., Москвичев В. В., Лепихин А. М. Вероятностные модели технологической дефектности сварных соединенийю - Красноярск : ВЦ СО АН СССР, 1988. - (Препр. АН СССР, Сиб. отд-ние, ВЦ; N 8.

23. Москвичев В. В., Доронин С. В. Оценка и оптимизация долговечности и надежности при ресурсном проектировании сварных конструкций // Заводская лаборатория. Диагностика материалов. - 1996. - № 3. - С. 38-42.

24. Stoer M., Wagner F. A simple min-cut algorithm // Journal of the ACM. - 1997. - Vol. 44, No. 4. - P. 585-591. - DOI: 10.1145/263867.263872. 